\title{
Kinetic Effects on the Currents Determining the Stability of a Magnetic Island in Tokamaks
}

\author{
E. Poli ${ }^{1}$, A. Bergmann ${ }^{1}$, F. J. Casson ${ }^{1}$, W. A. Hornsby ${ }^{1}$, A. G. Peeters ${ }^{2}$, \\ M. Siccinio ${ }^{1}$, D. Zarzoso ${ }^{1,3}$ \\ ${ }^{1}$ Max-Planck-Institut für Plasmaphysik, Garching, Germany \\ ${ }^{2}$ Department of Physics, University of Bayreuth, Bayreuth, Germany \\ ${ }^{3}$ PIIM, University of Aix-Marseille, Marseille, France
}

\begin{abstract}
The role of the bootstrap and polarization currents for the stability of neoclassical tearing modes is investigated employing both a drift kinetic and a gyrokinetic approach. The adiabatic response of the ions around the island separatrix implies, for island widths below or around the ion thermal banana width, density flattening for islands rotating at the ion diamagnetic frequency, while for islands rotating at the electron diamagnetic frequency the density is unperturbed and the only contribution to the neoclassical drive arises from electron temperature flattening. As for the polarization current, the full inclusion of finite orbit width effects in the calculation of the potential developing in a rotating island leads to a smoothing of the discontinuous derivatives exhibited by the analytic potential on which the polarization term used in the modelling is based. This leads to a reduction of the polarization-current contribution with respect to the analytic estimate, in line with other studies. Other contributions to the perpendicular ion current, related to the response of the particles around the island separatrix, are found to compete or even dominate the polarization-current term for realistic island rotation frequencies.
\end{abstract}

\section{Introduction}

There is a wide literature devoted to the tearing instability [1], and in particular to its nonlinear evolution [2] (see Ref. [3] and references therein for a recent overview; only a few relevant references are quoted below). This huge effort reflects on one hand the complexity of the underlying physics and on the other hand the experimental relevance of this subject. Although the first investigations were performed in the frame of a single-fluid MHD model, the importance of two-fluid $[4,5,6]$, kinetic $[7,8]$ and finite-Larmor-radius $[9,10,11]$ effects was recognized soon. In addition, several new features are introduced when toroidicity is included in the analysis. The most known examples are the effect of the favourable average curvature $[12,13]$, the neoclassical enhancement of the polarization current $[14,15]$ and the bootstrap drive $[16,17]$. These three contributions to the dynamics of magnetic islands, together with the contribution of the equilibrium current profile $\left(\Delta^{\prime}\right.$ term as in Ref. [2]), implemented in a so-called generalized Rutherford equation, are routinely exploited for the interpretation of experimental data (including active stabilization) $[18,19,20,21]$ and in the extrapolations to reactor-scale plasmas [22, 23, 24].

The goal of this paper is mainly to review the work on kinetic and toroidal effects on the stability of neoclassical tearing modes (NTMs) performed by the authors employing both a drift kinetic and a gyrokinetic approach in fully toroidal tokamak geometry. However, some new results concerning the polarization current are also included, and presented 
in Sec. 5. The ansatz employed here relies on the scale separation between transport time scales and island growth rate, so that both the width and the rotation frequency of the magnetic island are imposed and kept fixed, and the response of the plasma to the corresponding magnetic perturbation is determined. In this scheme, the feedback of this response on the island itself, which is accessible to reduced models based on a fluid and/or cylindrical or slab description, is therefore excluded. In the studies reported in this paper, on the other hand, toroidicity and kinetic effects are retained. Moreover, the specific focus is on the neoclassical contributions (bootstrap and neoclassical polarization currents), so that the role of turbulent fluctuations is disregarded. Some considerations on this issue can be found in Sec. 6. The inclusion of a toroidal kinetic description of the plasma, not bound to the ordering assumptions necessary to develop a tractable analytic theory, requires as a rule a numeric treatment. This has revealed a rich physics, which is often not included in the modelling of experimental observations. In some cases, like for the behaviour of the bootstrap current in small rotating islands, the numerical results can be explained in terms of known physical mechanisms. In other cases, in particular for the currents related to the motion of a magnetic island with respect to the surrounding plasma, like the polarization current, a complete understanding has not been achieved yet (although some aspects of the numerical modelling have been explained analytically) and requires further theoretical work. This appears particularly important, as the numerical results show a clear deviation from the behaviour expected on the basis of the available theoretical framework.

As stated above, the emphasis is here on the bootstrap and polarization contributions to the nonlinear tearing-mode evolution. Kinetic effects (finite-orbit-width effects and impact of the different response of passing and trapped particles) are shown to modify the island drive, related to the part of the parallel current flowing in phase with the island, see Sec. 2. The influence of kinetic effects on the island rotation (out-of-phase currents), which is more subtle $[10,15,25]$, is not considered at this level. A particularly important issue is the electrostatic potential associated with the presence of a magnetic island, in particular to its rotation. In numerical drift kinetic simulations, the electrostatic potential is not determined self-consistently and has to be prescribed according to analytic theory. On the other hand, in a gyrokinetic code this potential emerges self-consistently from the quasi-neutrality condition. This allows a check of the assumptions made in analytic theory. The basic physics underlying the determination of the electrostatic potential in the presence of an island is summarized in Sec. 2. The current analytic understanding of magnetic islands in toroidal plasmas employing a kinetic approach is largely based on the work of Wilson et al. [15], which is reviewed in Sec. 3. The approximations entailed in the theory are discussed. The results concerning the bootstrap current in small islands ${ }^{1}$ are presented in Sec. 4. It is shown, in particular, that the bootstrap drive of a NTM depends, in the phase where finite-orbit-width effects are important, on the rotation frequency of the island, being large if the island is rotating in the ion diamagnetic direction, and small if the rotation is in the electron diamagnetic direction. Sec. 5 is devoted to the investigation of the perpendicular current that vanish when flux-surface averaged, but contributes to the island dynamics since the related return current is in phase with the island. This contribution is usually called the polarization current. Here, this perpendicular current is shown to exhibit a complex behaviour, which is related also to the toroidal drift of trapped particles and alters the expected polarization-current behaviour for island rotation frequencies in an experimentally relevant range. The main results are summarized and conclusions are drawn in Sec. 6. The appendix offers a compact review of the numerical schemes (drift kinetic and gyrokinetic) employed in the simulations reported in the paper.

\footnotetext{
${ }^{1}$ In this paper, the words "small" or "large" applied to the magnetic-island size always refer to its width with respect to the typical ion-orbit width (gyroradius in slab geometry and banana width in toroidal geometry).
} 


\section{The electrostatic potential associated with the rota- tion of a magnetic island}

The evolution of a magnetic island is usually described by introducing a magnetic-flux perturbation of the form $\psi=\tilde{\psi} \cos \xi$, where $\xi$ is a helical coordinate along the island, and integrating Ampère's law across the tearing layer (where $\tilde{\psi}$ is taken nearly constant), projecting out the in-phase and out-of-phase components of the parallel current [26]. Matching the results to the outer linear region [2] one obtains

$$
\begin{aligned}
\frac{c}{4} \tilde{\psi} \Delta^{\prime} & =\oint \mathrm{d} \xi \int \mathrm{d} x J_{\|} \cos \xi \\
0 & =\oint \mathrm{d} \xi \int \mathrm{d} x J_{\|} \sin \xi .
\end{aligned}
$$

The stability parameter $\Delta^{\prime}$ is determined by the analysis of the outer region. The studies presented in this paper refer to the inner region, related to the right-hand side of the previous equations. In particular, we will concentrate on the contributions to the growth or damping of the magnetic island, as determined by Eq. (1). The rotation frequency of the island, which is determined by the out-of-phase currents flowing around the rational surface, Eq. (2), is treated in the following as a parameter.

A crucial quantity for our investigations is the electrostatic potential $\varphi$ connected with the rotation of a magnetic island with respect to the surrounding plasma. The basic behaviour of $\varphi$, in particular its scaling with island width and rotation frequency, can be understood in terms of the fluid picture explained in Ref. [27] (slab geometry, isothermal electrons, parallel ion dynamics neglected), which is summarized here for convenience. The fundamental equations are then

$$
\begin{aligned}
\frac{\mathrm{d} n}{\mathrm{~d} t} & =\frac{1}{e} \nabla_{\|} J_{\|} \\
E_{\|} & =-\frac{T_{e}}{e n_{0}} \nabla_{\|} n
\end{aligned}
$$

representing the electron continuity and parallel momentum balance (Ohm's law), respectively. In the presence of a magnetic island, the parallel gradient takes the form

$$
\nabla_{\|}=\mathbf{b} \cdot \nabla=\left.k_{\|} \frac{\partial}{\partial \xi}\right|_{\Omega},
$$

where $\Omega$ is a flux label for the perturbed magnetic surfaces and

$$
k_{\|}=-\frac{k_{\theta} x}{L_{s}},
$$

( $x$ is the distance from the rational surface, $k_{\theta}$ the poloidal wavenumber of the island and $L_{s}$ the shear length). The fundamental relation between density perturbation and electrostatic potential is postulated in the two opposite limits of an island width $W$ which is much larger or much smaller than the typical ion-orbit size $\rho$. Specifically, for $W \ll \rho$ an adiabatic ion response

$$
\delta n=-\frac{e \varphi}{T_{i}} n_{0}
$$

is assumed, while in the opposite limit $W \gg \rho$ the polarization equation

$$
\frac{c^{2}}{4 \pi e v_{A}^{2}}\left(1-\frac{\omega_{* i}}{\omega}\right) \frac{\mathrm{d}}{\mathrm{d} t} \nabla_{\perp}^{2} \varphi=\frac{1}{e} \nabla_{\|} J_{\|}
$$

is introduced. In this equation, $\omega_{* i}$ is the ion diamagnetic frequency with inclusion of the temperature-gradient contribution. It should be noted that Eqs. $(7,8)$ can be obtained 
taking the appropriate limits of a general expression for $\delta n$ (valid for arbitrary $\rho / W$ ) which can be derived from the gyrokinetic equation [10].

In the small-island limit, i.e. inserting Eq.(7), Eq.(4) becomes (in the whole derivation reported below, it is assumed that $\omega \gg \mathrm{d} \ln W / \mathrm{d} t)$

$$
\left.\left(-\omega+\omega_{* e}\right) \frac{\partial \psi}{\partial \xi}\right|_{x}-\left(1+\frac{1}{\tau}\right) c \nabla_{\|} \varphi=0 .
$$

Here, $\psi=-A_{\|}$and $\tau=T_{i} / T_{e}$. This equation can be immediately integrated exploiting $\nabla_{\|} x=-\left(k_{\theta} / B\right) \partial \psi /\left.\partial \xi\right|_{x}$ to give

$$
\varphi=\frac{\tau}{1+\tau} \frac{B}{c k_{y}}\left(\omega-\omega_{* e}\right)(x-h(\Omega)),
$$

The function $h(\Omega)$ arises as an integration "constant", cf. Eq. (5), chosen in such a way that the electric field vanishes at infinity (i.e. at distances from the island much larger than the island width) and is assumed to be flat $(\mathrm{d} h / \mathrm{d} \Omega=0)$ inside the island, see discussion below. The important feature of Eq. (9) for the investigations presented later is the dependence of the potential on the island rotation frequency in the form $\omega-\omega_{* e}$. Eq. (7) implies the following form for the density profile

$$
n=n_{0}(x)+\delta n=n_{0}^{\prime} x-\frac{1}{1+\tau}\left(1-\frac{\omega}{\omega_{* e}}\right) n_{0}^{\prime}(x-h(\Omega)) .
$$

For (small) islands rotating at the electron diamagnetic frequency, the perturbed density and the electrostatic potential are both zero, i.e. the density profile retains its unperturbed shape. For (small) islands rotating at the ion diamagnetic frequency, on the other hand, the first term in the density perturbation exactly (for $\tau=1$ ) cancels the background gradient and the density $n=n_{0}^{\prime} h(\Omega)$ is flat inside the island. It is noted that substituting Eq. (10) into Eq. (3) leads to a closed expression for the parallel current which can be used to infer the stability of the island, cf. Eq. (1).

A similar calculation can be performed in the large-island limit (see again Ref. [27] for details). In this case, the electrostatic potential becomes (to the lowest order in $\rho / W$ )

$$
\varphi=\frac{\omega B}{c k_{y}}(x-h(\Omega)) .
$$

The function $h(\Omega)$ has the same asymptotic behaviour as before. The density profile takes the form $n=n_{0}^{\prime} h(\Omega)$ independently of the rotation frequency. In the absence of sources and neglecting the transport across the island separatrix, the density can be assumed to flatten across the whole island, leading to the assumption $\mathrm{d} h / \mathrm{d} \Omega=0$ mentioned before. The neglect of cross-field transport is justified if the island width exceeds a critical width proportional to $\left(D_{\perp} / D_{\|}\right)^{1 / 4}[28]$. Similar considerations apply for the temperature profile. This choice for $h(\Omega)$, which is justified under the assumptions discussed above, is maintained also in the small-island limit. It is noted that the density in the large-island limit satisfies $\nabla_{\|} n=0$ independently of the island rotation frequency and Ohm's law reduces to the MHD constraint $E_{\|}=0$. The electrostatic potential arising in response to the inductive field associated with the island rotation leads to an $E \times B$ velocity which ensures that the plasma moves together with the magnetic island.

Two comments are in order here. First, the potential given by Eq. (11) has discontinuous derivatives at the island separatrix. This fact has significant consequences for the evaluation of the polarization-current term in the modelling of magnetic islands and is discussed later in Sec. 5. Second, the level of density flattening inside a rotating magnetic island is independent of the rotation frequency only if the island is significantly wider than the typical ion-orbit width. This condition is not satisfied in the early phase of development of a magnetic island [29]. The discussion above suggests that the neoclassical (bootstrap) drive could depend on the rotation frequency in that phase, if the results found in slab geometry turned out to hold also in toroidal geometry. This issue is discussed in Sec. 4 


\section{Neoclassical theory in the presence of a magnetic island}

The effort devoted to development of a toroidal kinetic theory of the tearing mode is very limited as compared to the body of publications in two-dimensional geometry. The reason for this is undoubtedly the difficulty of the analytic treatment, which is feasible only under particular assumptions. Here we follow Ref. [15], where a double small-parameter expansion of the distribution function is introduced in order to obtain a perturbative solution of the drift kinetic equation:

$$
f_{\alpha}=\left(1-\frac{q_{\alpha} \varphi}{T_{\alpha}}\right) f_{M \alpha}+g_{\alpha} \quad g_{\alpha}=\sum \delta_{\alpha}^{i} \Delta^{j} g_{\alpha}^{(i, j)}
$$

where $\Delta=W / r$, while $\delta_{e}=\rho_{p e} / W$ and $\delta_{i}=\sqrt{\epsilon} \rho_{p i} / W$ are defined in analogy to standard neoclassical theory ( $\rho_{p}$ is the thermal poloidal gyroradius and $\sqrt{\epsilon} \rho_{p}$ the thermal banana width; note however that for the ions $W \sim \rho_{p i}$ is assumed). Since $\delta_{e, i} \ll 1$, the theory is valid only in the "large island" limit. The electrostatic potential is determined from the assumption of density flattening inside the island and $E_{\|}=0$ and is thus given by, cf. Eq. (11),

$$
\varphi=\frac{\omega q}{m c}(\chi-h(\Omega)),
$$

where $m$ is the poloidal mode number, $q$ is the safety factor and $\chi$ is the poloidal flux. The lowest-order solution for $g$ is

$$
g_{\alpha}^{(0,0)}=\frac{q_{\alpha} f_{M \alpha}}{T_{\alpha}} \frac{q}{m c}\left(\omega-\omega_{* \alpha}\right)(\chi-h(\Omega))=\frac{q_{\alpha} \varphi}{T_{\alpha}} f_{M \alpha}-\frac{\partial f_{M \alpha}}{\partial \chi}(\chi-h(\Omega)),
$$

where the second form is a consequence of Eq. (13) and contains a term which cancels the adiabatic splitting in the ansatz of Eq.(12) and the second expresses the profile modification in the presence of a magnetic island (with corresponding flattening inside it). The nextorder term is related to the neoclassical fluxes and can be written

$$
g_{\alpha}^{(1,0)}=-\frac{I v_{\|}}{\Omega_{\alpha}}\left(\frac{\partial g_{\alpha}^{(0,0)}}{\partial \chi}+\frac{\mathrm{d} n / \mathrm{d} \chi}{n} f_{M, \alpha}\right)+h_{\alpha},
$$

where $I=R B_{t}$ is a flux function and $h_{\alpha}$ is an integration "constant" (independent of the poloidal angle $\theta$ ). This term is not only important in the determination of the bootstrap drive, but also enters directly the expression for the neoclassical polarization current, whose contribution to the island dynamics is derived from the part of the current perturbation which flux-averages to zero: Following again Ref. [15], the quasi-neutrality equation $\nabla \cdot \mathbf{J}=$ 0 reads (angular brackets denote flux-surface average)

$$
\nabla_{\|} J_{\|}=-\left\langle\int \mathrm{d}^{3} v \mathbf{v}_{d} \cdot \nabla g\right\rangle \simeq \frac{R B}{\Omega_{i}} Z e \int \mathrm{d}^{3} v v_{\|} \frac{\partial}{\partial \chi}\left\langle\frac{v_{\|}}{R q} \frac{\partial g}{\partial \theta}\right\rangle,
$$

where only the ions contribute to the right-hand side and the lowest-order non-vanishing term in expansion of $g$ is given by

$$
\left\langle\frac{v_{\|}}{R q} \frac{\partial g^{(1,1)}}{\partial \theta}\right\rangle=-\left\langle\frac{\mathrm{d}}{\mathrm{d} t} g_{i}^{(1,0)}\right\rangle-\left\langle v_{\|} \nabla_{\|} g^{(1,0)}\right\rangle+\ldots
$$

The dots denote terms which do not contribute to Eq.(16) and $g^{(1,0)}$ is given by Eq.(15). In the present framework, the two contributions on the right-hand side are of the same order, since the underlying ordering assumption for the time scale associated with the island rotation is $\omega \sim \omega_{*}$. Correspondingly, $\omega_{*} /\left(v_{\mathrm{th}, i} / R q\right) \sim \rho_{p i} / L_{n}$, while $k_{\|} v_{\mathrm{th}, i} /\left(v_{\mathrm{th}, i} / R q\right) \sim$ $W / L_{q}$, which are of same order if $L_{n} \sim L_{q}$, and $W \sim \rho_{p i}$ as stated before. To isolate 
the polarization current contribution, the limit $\omega>k_{\|} v_{\mathrm{th}, i}$ is invoked in Ref. [15] (thus neglecting the parallel ion dynamics as in the previous section), corresponding to $W<\rho_{p i}$. For this reason, the theory is strictly speaking valid only for $\sqrt{\epsilon} \rho_{p i}<W<\rho_{p i}$. Under these assumptions, a direct manipulation of Eq. (16) yields

$$
\nabla_{\|} J_{\|}=-\frac{R B}{\Omega_{i}} Z e \int \mathrm{d}^{3} v v_{\|} \frac{\partial}{\partial \chi}\left\langle\frac{\mathrm{d}}{\mathrm{d} t} g_{i}^{(1,0)}\right\rangle \approx-\nabla_{\perp} \frac{Z e}{\Omega_{p i}} \int \mathrm{d}^{3} v \frac{v_{\|}^{2}}{v_{\mathrm{th}, i}^{2}} f_{M i} \frac{\mathrm{d} \omega_{E}}{\mathrm{~d} t}
$$

$\left(\Omega_{p i}\right.$ is the ion cyclotron frequency calculated with the poloidal field and $\left.\omega_{E}=c E_{r} / B_{p}\right)$. The last step in Eq. (18) is achieved by substituting Eq. (15) and neglecting density and temperature gradients. It is recalled that in a fluid picture the neoclassical polarization current is enhanced by a factor $q^{2} / \epsilon^{2}$ with respect to its classical counterpart [14] and has the form $j_{\text {pol }}^{\text {nc }}=\left(e n / \Omega_{p i}\right) \mathrm{d} \omega_{E} / \mathrm{d} t$, which has the same structure as the right-hand side of Eq. (18) above. It is noted in passing that this expression makes immediately clear why the polarization current is expected to be a quadratic function of the island rotation frequency: $\omega_{E}$ is proportional to $\omega$, as it involves the gradient of the electrostatic potential, and its time variation scales again with $\omega$. In the presence of background gradients, the polarization current is proportional to $\omega\left(\omega-\omega_{* i}\right)[27]$.

The above estimate (18) is valid only in the so-called collisional regime for the island polarization current, in which also the passing ions contribute to the perpendicular current. If only the trapped particles are involved, the neoclassical enhancement is $q^{2} / \sqrt{\epsilon}$. In the derivation above, this is described by the function $h_{\alpha}$ in Eq. (15), which cancels the contribution of the passing particles to the integral in Eq. (18). The transition between low and high collisionality regimes is briefly discussed at the end of Sec. 5. It is noted for later reference that the function $h_{i}$ as derived in Eq. (58) of Ref. [15] contains a resonant denominator, the resonance corresponding to an island rotation frequency matching the parallel streaming of the passing ions. Neglecting the parallel streaming against the island rotation, as done by assuming $\omega>k_{\|} v_{\mathrm{th}, i}$, implies that this resonant behaviour is discarded. The point is briefly addressed in Sec. 5.1.

\section{Bootstrap current}

The flattening of the pressure profile inside a magnetic island of sufficient width leads to a loss of bootstrap current. This perturbation is destabilizing for standard positive-shear scenarios and leads to the well-know neoclassical drive, which is believed to be the usual cause of tearing mode destabilization in reactor-grade tokamak plasmas. The bootstrap drive in the generalized Rutherford equation is found to depend inversely on the island width $W[16,17]$. Regularizations of the related divergence for vanishing $W$, due to finite perpendicular transport [28] (see above) or finite ion-orbit width [29, 30], have been proposed and included in the modelling [31]. Since the bootstrap current contribution to the generalized Rutherford equation has been derived in the large-island limit, it does not entail any dependence on the rotation frequency. In this section, finite-orbit effects are reviewed and the role of the rotation frequency is discussed in light of the results of Sec. 2 .

The first investigations of the impact of the overlap between trapped-ion orbits and a magnetic island on the neoclassical nonlinear drive of the tearing mode were limited to the ion contribution to the bootstrap current and were performed solving numerically the drift kinetic equation for the ions in the presence of a prescribed, static magnetic island [29, 30], cf. App. A. The assumption of large island width (much larger than the thermal ion banana width, $\sqrt{\epsilon} \rho_{p i} / W \ll 1$ ) employed in the analytic theory presented in Sec. 3 becomes unnecessary in the case of a numerical approach. This early work shows that, for island widths comparable to the ion banana width, the (ion) bootstrap perturbation is reduced, and vanishes for $W \lesssim \sqrt{\epsilon} \rho_{p i}$, implying a corresponding reduction of the neoclassical drive. A further result is that a threshold of the bootstrap drive related to finite-ion-orbit-width effects naturally leads to a scaling of $\beta_{p}$ at the onset of the 
tearing mode with the normalized poloidal gyroradius $\rho_{p i}^{*}$, in agreement with experimental observations [32]. This study was later extended to (imposed) rotating magnetic islands and to the calculation of total bootstrap current [33]. The electron contribution to the bootstrap current is included through a two-step scheme. The drift kinetic equation for the ions is solved first, assuming that their collisions with electrons can be neglected. In this way the parallel ion flow profile $u_{i, \|}$ is obtained. In a second step, the drift kinetic equation is solved for the electrons, including $u_{i, \|}$ as a shift in the background electron distribution function $f_{0 e}$; the deviation $\delta f_{e}$ from the background is computed including both electronelectron and electron-ion collisions. Since $f_{e 0}$ produces a parallel electron current that exactly cancels the ion current, the total bootstrap current can be calculated directly as $v_{\|}$-moment of $\delta f_{e}$. A difficulty arises from the fact that, in the case of small islands, the ions are found to respond adiabatically to the potential, as described in Sec. 2. The electrostatic potential is imposed in the drift kinetic simulations according to analytic theory. In the case of a small island, prescribing $\varphi$ according to its large-island form, Eq.(11), can lead to a violation of quasi-neutrality. The reason is that finite-orbit-width effects are negligible for electrons, so that the electron density and temperature profiles tend to be flat also inside small islands (apart from the contribution of trapped particles, see below; crossfield transport is not considered at this stage), independently of the rotation frequency. The degree of flattening of the ions depends instead on the potential and thus on the island rotation frequency. In particular, the electrostatic potential is such that nearly unperturbed density profiles are expected for islands rotating at the electron diamagnetic frequency, as explained in Sec. 2. A form of the electrostatic potential consistent with quasi-neutrality for small island widths can be derived analytically in the frame of the drift kinetic analysis of Sec. 3, recalling the fact that the expansion of the distribution function as in Eq. (12) still holds for electrons. Imposing an adiabatic response for the ions, $n_{i}=n_{0}\left(1-e \varphi / T_{i}\right)$, and equating the ion density to the electron density resulting from $f_{e}$ leads to lowest order to [33]

$$
\varphi_{\text {small }}=\frac{\tau}{1+\tau} \frac{\omega-\omega_{* e}}{\omega} \varphi
$$

where $\varphi$ on the right-hand side is given by Eq. (13). This result is equivalent to the slabgeometry expression of the electrostatic potential in small islands (9). When substituted into Eqs. $(12,14)$, Eq. (19) leads to electron density profiles that follow the ion ones, being nearly unperturbed for $\omega=\omega_{* e}$ and flat inside the island for $\omega=\omega_{* i}$. The implementation of Eq. (19) in numerical drift kinetic simulations employing the two-step scheme described above enables the determination of the total bootstrap current in small rotating islands. The results are shown in Fig. 1. 


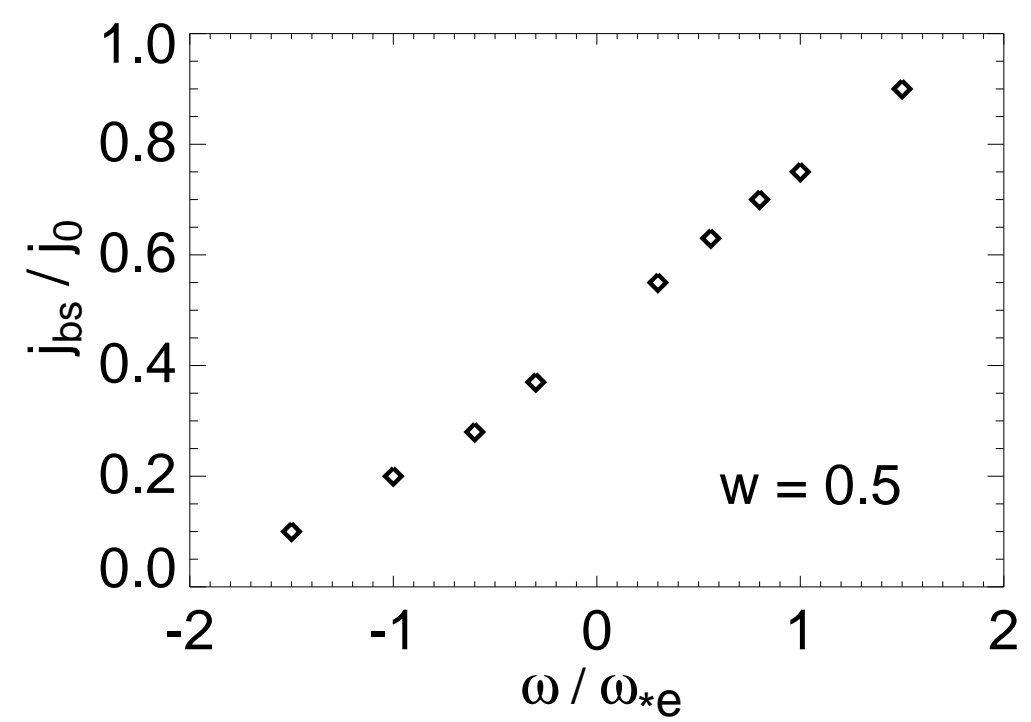

Fig. 1. Bootstrap current inside a small island (half the thermal ion banana width), normalized to its unperturbed value, as a function of the island rotation frequency. The electrostatic potential in these drift kinetic simulations has been specified according to Eq. (19). The neoclassical drive (proportional to the bootstrap-current perturbation) decreases as the rotation frequency is moved from the ion direction to the electron direction [Reprinted with permission from A. Bergmann, E. Poli, A. G. Peeters, Phys. Plasmas 16, 092507. Copyright 2009, American Institute of Physics.].

It can be clearly seen that the bootstrap current perturbation inside an island whose width is half the thermal ion banana width clearly depends on the island rotation frequency. For values of $\omega$ of the order of the ion diamagnetic frequency, the density profile is substantially flat and the residual bootstrap current found inside the island is due a finite ion temperature gradient. In the other limit $\omega \simeq \omega_{* e}$, the bootstrap current is close to its unperturbed value, the residual perturbation being related to a flat electron temperature.

The previous results clearly show that for small islands the bootstrap drive depends on the rotation frequency, being strong when the islands rotate in the ion direction and weak when they rotate in the electron direction. These conclusions are based on a potential given by Eq.(19), which is calculated analytically assuming an adiabatic ion density perturbation and imposing quasi-neutrality. In the drift kinetic simulations discussed above, this potential is an input for the simulations and is not computed self-consistently. To test the validity of this model, gyrokinetic simulations have been performed [34]. In this gyrokinetic setup, the magnetic perturbation generating the island (width and rotation frequency) is imposed, like in the drift kinetic simulations, but the potential is calculated self-consistently solving numerically the gyrokinetic Poisson equation. While the fine structure of $\varphi$ around the separatrix is found to be different from the non-differentiable behaviour of the analytic solution, as discussed in the next Section, the picture presented above is fully reproduced (Fig. 2), thus confirming the conclusions derived on the basis of drift kinetic calculations. In particular, the electrostatic potential inside a small magnetic island is found to be flat (causing no electric field) around the electron diamagnetic frequency, whose value is $\omega_{* e} \simeq 0.06$ in these simulations (frequencies are normalized to $\left.v_{\mathrm{th}, i} / q R\right)$. As the island becomes larger, the island rotation frequency corresponding to flat potential moves towards $\omega=0$. In these simulations, the thermal ion banana width is about $3 \rho_{i}$. A further discussion is reported in Ref. [35]. 

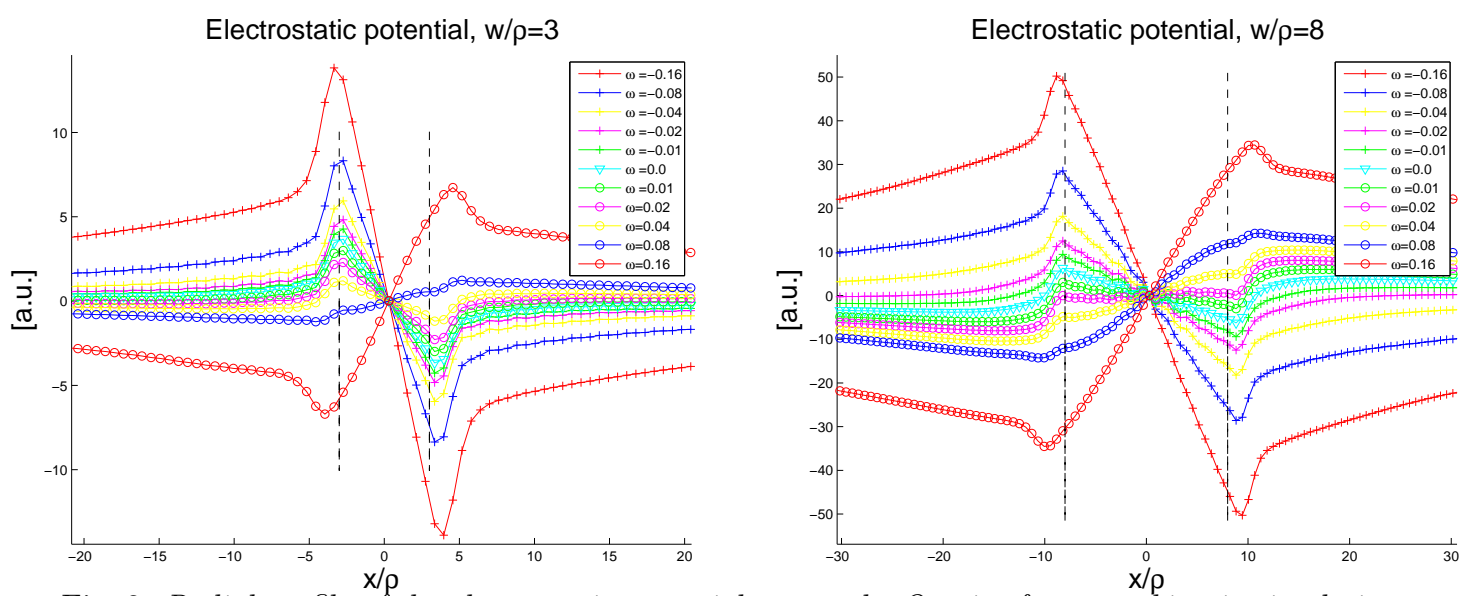

Fig. 2. Radial profile of the electrostatic potential across the O-point from gyrokinetic simulations of a rotating island for two different island widths, $W / \rho=3$ (left) and $W / \rho=8$ (right). The equilibrium diamagnetic frequency is in normalized units $\omega_{* e}=0.06$.

The next question to be addressed is whether the same findings should be expected in the presence of turbulent transport. Very recently, gyrokinetic simulations of magnetic islands in the presence of ion-temperature-gradient-driven turbulence [36] have shown that, if the island is not too big for the pressure to be flattened by the usual parallel streaming along the perturbed field lines, the dependence of the density gradient inside the island on the rotation frequency discussed above is reproduced. Further investigations are still necessary to confirm this behaviour in the presence of different kinds of turbulence (e.g. due to trapped-electron modes) and clarify possible modifications of the underlying physics with respect to the picture outlined in this Section.

It should be finally noted that turbulence simulations in the presence of a static island, supported by a simpler kinetic model, have also revealed that, if the trapped-particle population inside the island is replenished by the perpendicular transport on a time scale comparable to or exceeding the detrapping time, a finite pressure gradient and a finite bootstrap current are maintained [37]. This process leads to a critical magnetic island size below which the bootstrap drive becomes ineffective, which can be expressed as $w_{c}=$ $\sqrt{2 \epsilon D_{\perp} / \nu_{e i}}$, where $\nu_{e i}$ is the electron-ion collision frequency (for typical parameters of ASDEX Upgrade, for instance, this corresponds to a critical width of the order of $1 \mathrm{~cm}$, thus comparable to other threshold mechanisms discussed in the literature). This effect is included in the simulations of Ref. [36] mentioned above, which were performed at realistic collisionality. It is remarked that the impact of the island rotation was not considered in Ref. [37].

\section{$5 \quad$ Polarization current and beyond}

The effect of the bootstrap current perturbation on the island stability results from a fluxsurface-averaged parallel current. However, also currents that vanish on average along a flux surface can contribute to the growth or the damping of an island, if their phase matches that of the island. A contribution associated to the rotation of the island with respect to the surrounding plasma is the polarization current [38]. If stabilizing, it would represent a further threshold mechanism at small island widths. Due to its dependence on $W\left(\sim 1 / W^{3}\right)$, which leads to a sharp threshold in the modelling, and the predicted dependence of the critical $\beta$ at the mode onset with $\rho^{*}$, this term has been invoked to explain experimental data $[39,40]$. The sign of this contribution, however, is a quadratic function of $\omega$, see Sec. 3. For this reason, an assessment of its role is tightly linked to the problem of determining the rotation frequency of the island, which is very subtle, see the illuminating discussion by Mikhailovskii [41], and is still far from being solved under 
realistic tokamak conditions. Moreover, the magnitude of the contribution is related to the derivatives of $h(\Omega)$, which are discontinuous around the separatrix in the model described in Secs. 2,3. Finally, a toroidal kinetic modelling reveals a richer physics, mostly related to the presence of trapped particles.

The derivation of the polarization-current contribution to the island dynamics in the frame of neoclassical theory has been sketched in Sec 3. Before turning to further analytic and numerical results obtained in toroidal geometry, we recall that the polarization current contains also a "classical" contribution, due to the varying electric field experienced by the ions along a gyro-orbit. As discussed in Sec. 3, the neoclassical contribution, due to the varying electric field along the ion banana orbit, is larger by a factor between $q^{2} / \sqrt{\epsilon}$ and $q^{2} / \epsilon^{2}$, depending on collisionality (see Refs. [15, 42] for a detailed physical picture). Strictly speaking, the polarization current is a perpendicular current mainly carried by the ions, since it is an inertial effect. The parallel current contributing to the nonlinear evolution of the tearing mode arises to preserve quasi-neutrality and is carried by the electrons, which are much more mobile. In numerical simulations, the detection of the perpendicular ion flow is easier than that of the parallel closure current, as the parallel flow is dominated by the neoclassical $c E_{r} / B_{p}$ component. Moreover, in drift kinetic simulations $[43,42]$ the electrons are not traced. In order to isolate the role of the electric field associated with the island rotation, flat density and temperature profiles are imposed in the results presented below (for this reason "ion diamagnetic" and "electron diamagnetic" direction refer in the following to the direction of the diamagnetic flow "if there were" a corresponding gradient). In the absence of pressure gradients, the polarization current is expected to scale with the island rotation frequency simply as $J_{\text {pol }} \propto \omega^{2}$.

\subsection{Drift kinetic results}

In drift kinetic simulations performed imposing the electrostatic potential according to Eq. (13), the perpendicular ion flow turns out to deviate from a simple quadratic dependence, even if the island width is much larger than the thermal ion banana width, so that the ordering (12) adopted in the analytic theory discussed in Sec. 3 is valid. Instead of two degenerate zeros at $\omega=0, J_{\text {pol }}^{\text {neocl }}$ is found to cross the $x$-axis at four distinct points, see Fig. 3. A first possible explanation for this behaviour could be a contribution from the parallel streaming of the passing ion, which is of the same order as that leading to the polarization current, but has been neglected invoking the limit $W<\rho_{p i}$ as discussed above. However, no contribution from $v_{\|} \nabla_{\|} g^{(1,0)}$ can be expected around $\omega=0$, since the integrand is odd in $v_{\|}$, cf. Eqs. (15-17). When the parallel streaming is retained, a resonant denominator appears in the corresponding distribution function, as mentioned in Sec. 3. The right-hand side of Eq. (17), however, provides a numerator in the expression of the perpendicular current, which to leading order cancels the resonance [44]. As a consequence, no qualitative deviation from the polarization-current picture term arises from the parallel-streaming contribution. Numerical simulations confirm this behaviour for both the distribution function and of the perpendicular current. For this reason, the ion parallel streaming was ruled out in Ref. [44] as a possible cause for the deviation of the perpendicular ion flow from the expected quadratic behaviour. However, a more rigorous treatment of the resonant denominator would be needed to ascertain its overall role in the island dynamics, in particular in relation to the out-of-phase currents leading to the island rotation. 


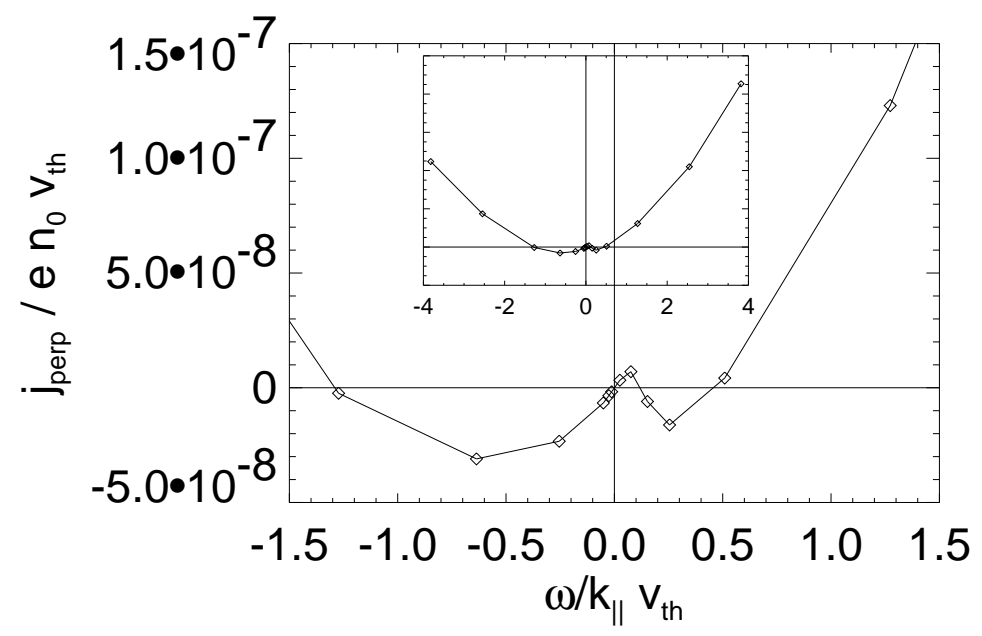

Fig. 3. Perpendicular current (averaged over a helical half-period of a magnetic island), as a function of the island rotation frequency. Results of drift kinetic simulations for a large island, about 10 times larger than the thermal ion banana width [Reprinted with permission from E. Poli, A. Bergmann, A. G. Peeters, L. Appel, S. D. Pinches, Nucl. Fusion 45 , 384 (2005).].

A second resonance, related to the matching between the toroidal precession frequency of trapped particles $\omega_{t p}$ and the toroidal rotation of the island, plays an important role in this respect $[43,42]$. This physics is not captured by the ordering employed in Sec. 3 , since the island is ordered there $\omega \sim \omega_{*} \sim \Delta$. To describe the resonance with the toroidal precession of trapped ions, a different ordering, $\omega \sim \delta \Delta$ has been introduced in Ref. [44] as an ansatz for a perturbative treatment of the drift kinetic equation along the line of what discussed in Sec. 3. In this case, the function $h_{i}^{(1,0)}$ is found to vanish in the passing domain and takes the form

$$
h_{i}^{(1,0)}=-\frac{m}{q} \frac{\omega_{t p}}{\omega+\frac{m}{q}\left(\omega_{E}+\omega_{t p}\right)} \frac{q_{i} \varphi}{T_{i}} F_{M}
$$

in the trapped domain. For $\omega$ around zero, this leads to a current scaling linearly with $\omega$, since $\varphi \propto \omega$, with a more complex pattern around the resonance, where slower particles cross the perturbed flux surfaces in one direction and faster particles in the opposite direction. A finite perpendicular current arises, which is due to the action of the toroidal electric field associated to the island motion on the equilibrium magnetic precession. For this reason, this contribution was dubbed "precessional current" in Ref. [44]. It is interesting to note that the toroidal precession frequency is (at least for thermal particles) usually comparable to or lower than the collision frequency, so that collisions effectively resolve the resonance. The parallel closure current associated to the precessional current can be calculated and a corresponding contribution to the generalized Rutherford equation can be explicitly derived. The dependence of the perpendicular ion current found in drift kinetic simulations on $\omega$ displayed in Fig. 3 can be explained in terms of a competition between polarization and precessional contributions, where the positive, nearly quadratic behaviour at higher frequencies can be ascribed to the polarization current while the precessional current has an opposite sign for islands rotating in the electron direction (negative in Fig. 3) or for islands rotating in the ion direction at a frequency larger than the toroidal precession frequency of the bulk (the exact point at which the precessional current reverses its sign for $\omega>0$ depends on the collision frequency, since collisions tend to act stronger on slower particles). Around $\omega=0$, the linear scaling mentioned above is found. The parallel current ensuring quasi-neutrality can be calculated. Stabilizing contributions correspond to negative values of $J_{\perp}$ in Fig. 3 . It is stressed that the modification with respect to a pure polarization scaling extends to frequencies of the order of the ion parallel streaming $k_{\|} v_{\text {th }, i}$. According to the discussion in Sec. 3, for an island size of the order of the poloidal ion gyroradius, the diamagnetic frequency would be of the order of $k_{\|} v_{\mathrm{th}, i}$ for $L_{n} \simeq L_{q}$, 
so that $k_{\|} v_{\mathrm{th}, i}$ can be taken as a proxy for the diamagnetic frequency, which is zero in the simulations. The deviation from the quadratic scaling discussed above, therefore, covers a range of frequencies which is experimentally relevant (see e.g. the discussion in Ref. [3] for a discussion of the expected island rotation frequency).

It is of particular interest (also for comparison with the gyrokinetic simulations presented in Sec. 5.2) to examine the radial profiles of the perpendicular ion current, as obtained from the drift kinetic simulations presented here, for frequencies at which the polarization current start to be detectable as a quadratic signal. In these profiles, obtained as an average value over a helical half-period of the island, two different behaviours can be discerned.
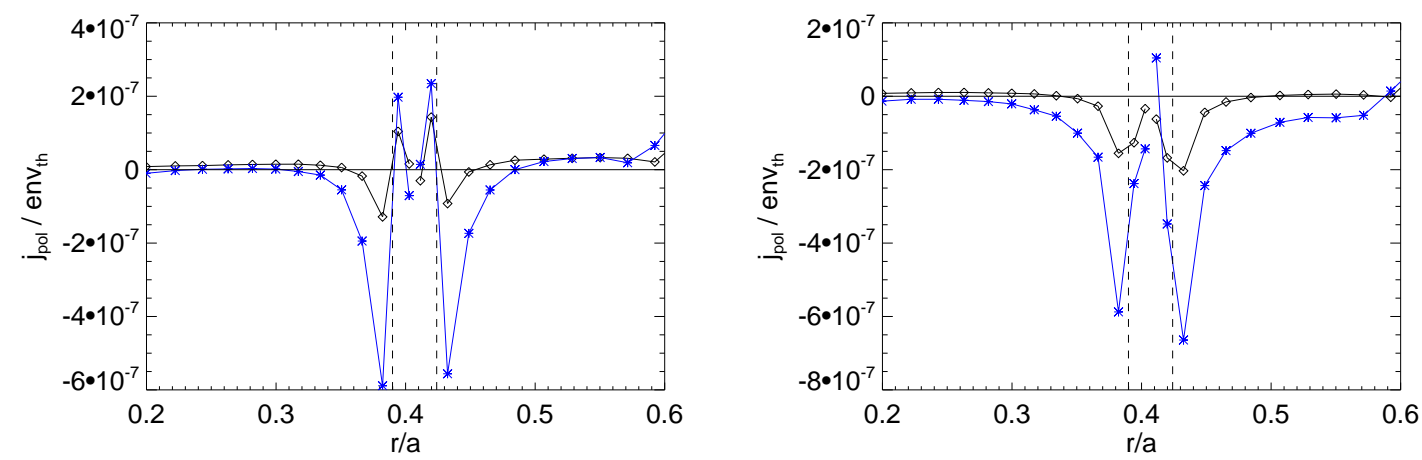

Fig. 4. Radial profile of the perpendicular ion flow (averaged over a helical half-period of a magnetic island) for two different island rotation frequencies, corresponding to $\omega / k_{\|} v_{\text {th,i }}=1.3$ (black diamonds) and $\omega / k_{\|} v_{t h, i}=2.6$ (blue stars). Left: Rotation in the ion diamagnetic direction (negative $x$-axis in Fig. 3). Right: Rotation in the electron diamagnetic direction (positive $x$-axis in Fig. 3). Two peaks, one scaling linearly and one scaling quadratically with $\omega$ can be identified just inside resp. outside the island separatrix (dashed black vertical lines).

This is particularly evident for islands rotating in the ion diamagnetic direction, left panel of Fig. 4. Just inside the island separatrix, a peak scaling linearly with $\omega$ can be identified, while the peak outside the island scales quadratically. The peaks have opposite sign. Consistently, for islands of similar frequency (in absolute value) rotating in the electron direction, the inner peak proportional to $\omega$ has an opposite sign than for islands rotating in the ion direction, while the outer peak proportional to $\omega^{2}$ has the same sign. A velocity-space analysis reveals that, while the outer ("polarization") peak is dominated by the contribution of the trapped particles, the inner linear peak is determined also by the radial excursion of the passing ions. This two-peak behaviour is recovered in gyrokinetic simulations, see below, where the electrostatic potential is computed self-consistently with the particle response. This complex current pattern, which still has to be fully understood, is expected to modify the sign of the return parallel current around the island separatrix and hence the stability of the tearing instability.

Before turning to gyrokinetic simulations, it should be mentioned here that the transition between low and high collisionality regimes $[15,45]$ for the polarization current has been also addressed by means of drift kinetic simulations $[43,46]$. In particular, the simulation results are seen to agree with the curves obtained by Mikhailovskii et al. [47]. In these simulations, access to high-collisionality regime (high values of $\nu / \omega$ ) could not be achieved simply by reducing $\omega$, since the perpendicular current is then dominated by the precessional contribution described above. On the other hand, increasing the collision frequency for values of $\omega$ corresponding to dominant polarization current often leads to a crossing of the banana/plateau regime, so that the full polarization current enhancement expected in the high-collisionality regime (proportional to $q^{2} / \epsilon^{2}$, as mentioned above) was usually not achieved in our simulations. See Ref. [46] for a detailed account. 


\subsection{Gyrokinetic results}

In gyrokinetic simulations, the electrostatic potential linked to the rotation of the island with respect to the surrounding plasma is calculated self-consistently from the gyrokinetic Poisson equation, cf. also App. A. This smooths out the non-differentiable behaviour of $\varphi$ across the island separatrix. The adiabatic ion response across the island separatrix is seen to contribute to the profile of $\varphi$ [34] (a similar effect had been investigated in relation to trapped fast-ion response [48]). On the other hand, an option has been added in the code GKW to allow the replacement of the self-consistent potential with an analytically imposed one [34], taken to be of the form given by Eq.(13). This enables a direct comparison of the importance of the electric-field discontinuity. A larger degree of smoothness is expected to lead to a smaller polarization current. This topic has been addressed also in previous work, e.g. through numerical integration of the equation for $\varphi$ [49], through a smoothing of $h(\Omega)$ [50], or a finite cross-field diffusion [51]. As a result, a substantial reduction of the ion polarization current is found.
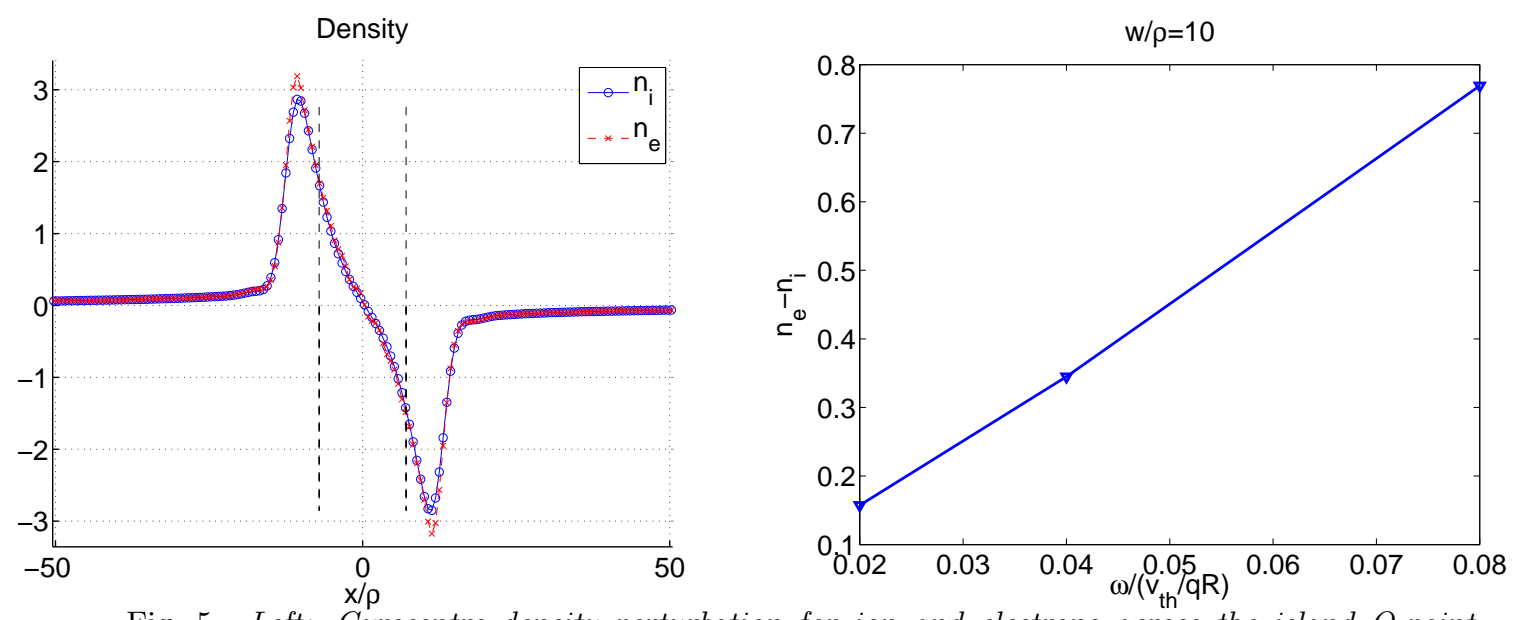

Fig. 5. Left: Gyrocentre density perturbation for ion and electrons across the island O-point (island width $W=10 \rho_{i}$ ). The difference represents the polarization density and is concentrated in the peak region just around the island separatrix. Right: The difference between electron and ion gyrocentre density as a function of the frequency, exhibiting the expected linear scaling.

We address the classical polarization current first. Fig. 5 shows a typical profile across the island O-point of the gyrocentre-density perturbation for an island of width $W=10 \rho_{i}$ rotating in the electron diamagnetic direction. The difference between the gyrocentre densities is the so-called polarization density $\delta n_{\text {pol }}$, which exhibits the same linear dependence on $\omega$ as the electrostatic potential, as expected. The classical polarization current is computed as

$$
J_{\text {pol }}^{\mathrm{cl}}=\int\left\langle\mathbf{v}_{E}\right\rangle \cdot \nabla \Omega \tilde{f}_{i}(\mathbf{R}) \mathrm{d}^{3} v-\int\left\langle\mathbf{v}_{E}\right\rangle \cdot \nabla \Omega \tilde{f}_{e}(\mathbf{R}) \mathrm{d}^{3} v
$$

with $\tilde{f}_{i, e}(\mathbf{R})$ denoting the (perturbed) gyrocentre distribution functions of ions and electrons, respectively, and the angular brackets the gyroaverage. A surface plot of $J_{\text {pol }}^{\mathrm{cl}}$ is shown in Fig. 6 (left) and reproduces the behaviour expected from the theory, cf. Fig. 1 of Ref. [42]: the (perpendicular) polarization current is concentrated in a narrow layer around the separatrix and has a $\sin \xi$ parity along the island, being zero in the regions at the level of the $\mathrm{O}$ and $\mathrm{X}$-point. 

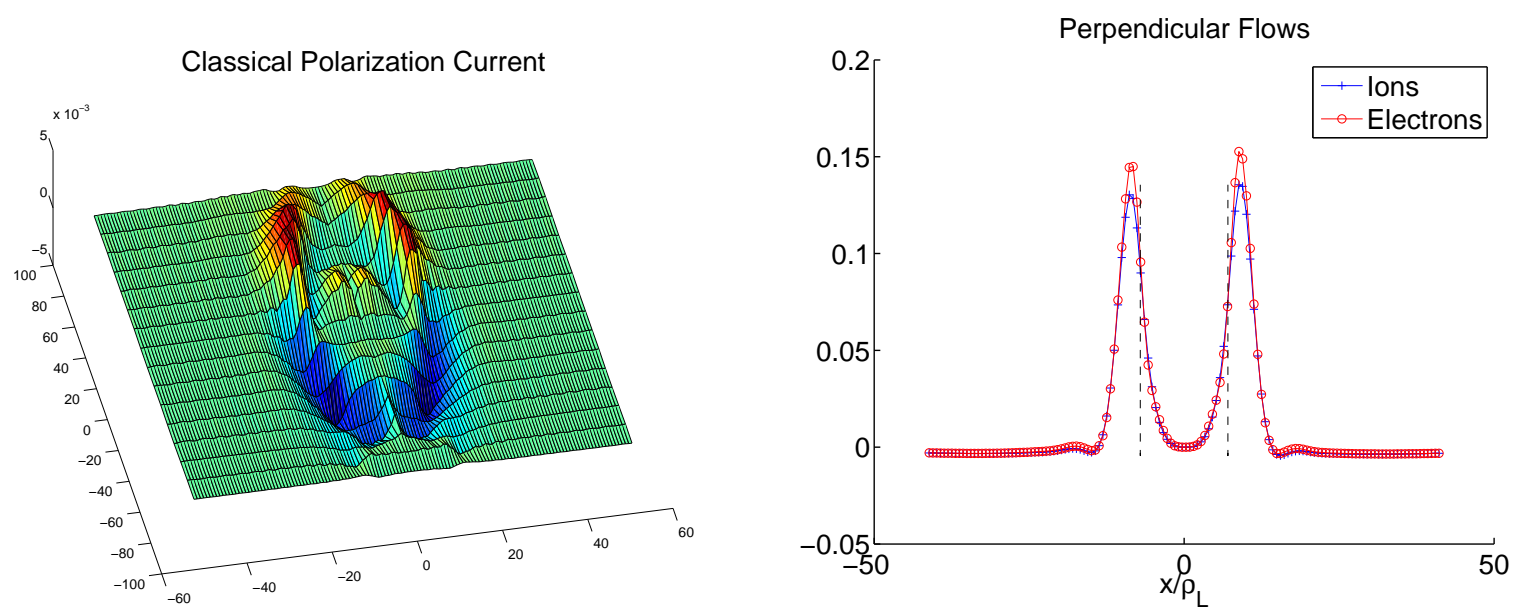

Fig. 6. Left: Surface plot of the classical polarization current. Right: Ion and electron gyrocentre flows, representing the two contributions to Eq. (21).

The $E \times B$ flow of the gyrocentres for electrons and ions is displayed in Fig. 6 (right), where a radial cut half-way between $\mathrm{O}$ and $\mathrm{X}$-point has been taken. A difference is seen to arise in the region around the island separatrix, where a finite polarization density is present. A scan in $\omega$ confirms that these flows scale quadratically, as expected.

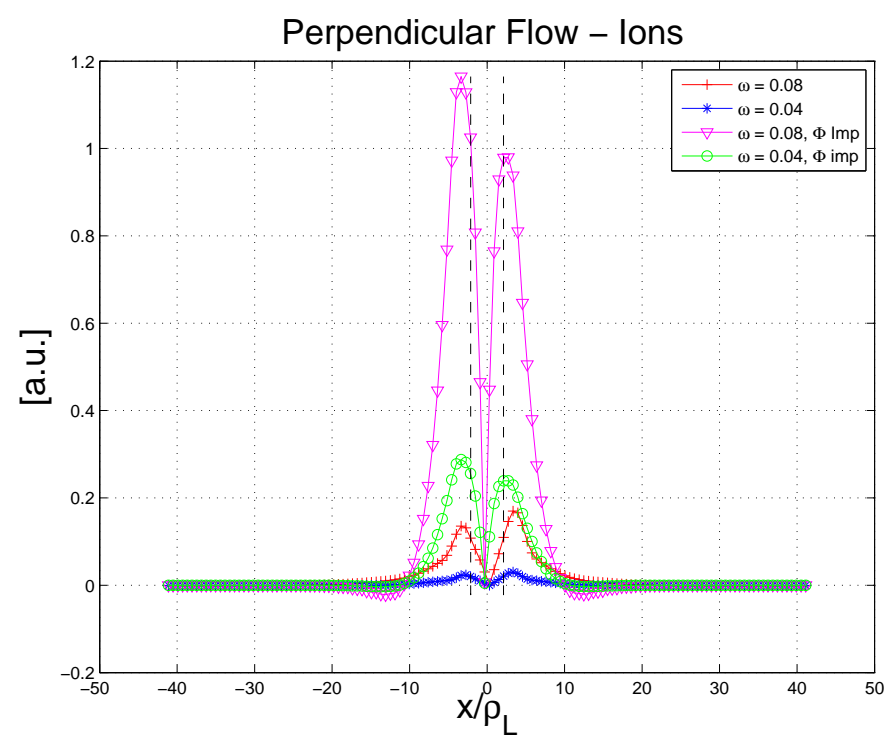

Fig. 7 Perpendicular classical ion flow, i.e. the first term on the right-hand side of Eq. (21), from gyrokinetic simulations with a potential imposed according to Eq. (13) (green circles and magenta triangles) or with self-consistent potential (red crosses and blue stars), for two different values of the island rotation frequency and $W=3 \rho_{i}$.

Fig. 7 shows a comparison between the classical gyrocentre fluxes with self-consistent and imposed electrostatic potential for a simulation with $W=3 \rho_{i}$, i.e. for an island width approximately equal to the thermal ion banana-orbit width. The frequencies have been shown in order to be of the order of the diamagnetic frequency if realistic gradient lengths were taken. The quadratic dependence on $\omega$ is evident also here. More importantly, the perpendicular gyrocentre flow originating from the imposed analytic potential produces a significantly higher flow than in the presence of a self-consistent potential. Note that, although an analytic potential obtained in the large-island limit (13) is not justified in the limit of islands of the same size as the ion orbit width, the polarization-current contribution to the generalized Rutherford equation as usually employed in the modelling is based on Eq. (13). More detailed analytic and numerical work is needed to quantify the entity of 
this effect in comparison to analytic theory.
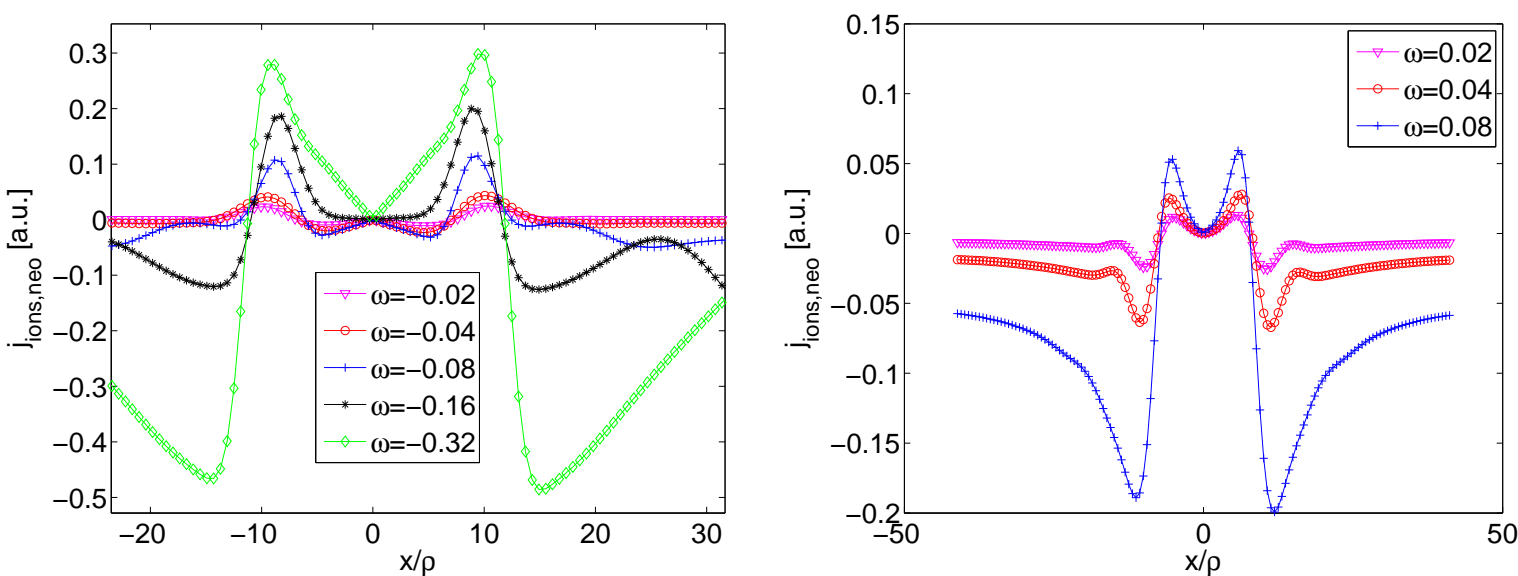

Fig. 8 Neoclassical ion flow profile through the island O-point from gyrokinetic simulations for and island rotating in the ion direction (left) and in the electron direction (right). Island width $W=10 \rho_{i}$

The neoclassical polarization current (or better the neoclassical perpendicular ion flow) originating from the island is calculated as $J_{i, \mathrm{pol}}^{\text {neocl }}=\int \mathbf{v}_{D i} \tilde{f}_{i}(\mathbf{R}) \mathrm{d}^{3} v$, where $\mathbf{v}_{D}$ is the magnetic drift velocity.

In Fig. 8, the radial neoclassical ion flow corresponding again to a cut half-way between O- and X-point is shown for different island rotation frequencies. For the parameters employed in these simulations, the ion parallel streaming (normalized to $v_{\mathrm{th}, i} / q R$ ) is in the range $k_{\|} v_{\mathrm{th}, i} \simeq 0.04$, where the parallel wavevector (6) is evaluated at a distance $x=W$ from the rational surface. The island width is $W=10 \rho_{i}$. For islands rotating in the ion diamagnetic direction (negative frequencies in these gyrokinetic simulations), a clear peak around the island separatrix can be observed, accompanied at low frequencies by a weaker peak of opposite sign inside the island. The peak at the island separatrix scales nearly linearly with the island frequency $\omega$. Outside the island, a peak of opposite sign, almost invisible at frequencies of the order of $k_{\|} v_{\mathrm{th}, i}$, develops for $\omega \gtrsim 0.1$. For islands rotating in the electron diamagnetic direction, the peak at the separatrix and the external peak are in the same direction and exhibit an intermediate scaling (between linear and quadratic) with $\omega$ at frequencies of the order of $k_{\|} v_{\mathrm{th}, i}$.

Some similarities with the picture emerging from the drift kinetic simulations presented in Sec. 5.1 can be observed. In particular, at frequencies of the order of $k_{\|} v_{\mathrm{th}, i}$, there is no clearly visible polarization-current signal (exhibiting a quadratic scaling with $\omega$ for flat pressure) in the perpendicular ion flow. Such a signal becomes instead dominant at higher frequencies. It is recalled that the polarization current is predicted to have a stabilizing effect on the island evolution for frequencies in the range between 0 and the ion diamagnetic frequency [52, 49]. Moreover, the two-peak structure described in Sec. 5.1 is clearly confirmed also by gyrokinetic simulations.

On the other hand, the gyrokinetic simulations presented here leave still some important questions open. One is connected to the precessional current described in Sec. 5.1. The frequency range around the toroidal precession frequency of trapped particles has not been considered in these simulations. Preliminary investigations show the expected resonance in velocity space, but the overall effect (including also the electrons, which have not been considered in the drift kinetic simulations) should be still investigated in detail. A second point to be addressed in detail is the specific impact of a potential satisfying quasineutrality also around the separatrix (where the assumption $E_{\|}=0$ of analytic theory fails). 


\section{Conclusions}

The dynamics of the tearing mode in tokamak plasmas involves an interplay of processes occurring on several disparate time and space scales. This makes the problem extremely complex both theoretically and computationally. For this reason, in the approach to a full theoretical understanding of the underlying physics, the main line of research is based on self-consistent models with reduced physical scope (fluid equations and/or simplified geometry). The results presented in this paper follow a complementary philosophy. Kinetic and geometrical (toroidal) effects are fully retained and the response of the system to a given magnetic perturbation is computed, but the feedback of this response on the evolution of the mode is discarded. The width and the rotation frequency of the island are treated as parameters. These studies are based mainly on a numerical approach, that makes the assumptions needed in analytic treatments $[17,15]$ unnecessary. Still, analytic works represent the starting point for any attempt at a more complete description based on more general physics assumptions.

In this paper, the neoclassical contributions to the evolution of a magnetic island due to bootstrap and polarization currents have been investigated employing both a drift kinetic and a gyrokinetic approach. A particularly important role is played by finiteorbit-width effects around the island separatrix, where the ions respond adiabatically, i.e. the perturbed ion density follows the electrostatic potential. Since the potential has a different slope inside the island depending on the island rotation frequency, also the degree of density flattening is a function of $\omega$. This, in turn, determines the level of neoclassical (bootstrap) drive to be expected in a small rotating island, which turns out to be large for islands rotating in the ion direction and vanishing for islands rotating at the electron diamagnetic frequency. The perpendicular flows in the presence of a rotating island have been also investigated, choosing a flat background pressure to isolate the effect of the island electric field. The classical polarization current, as determined from gyrokinetic simulations, shows a reduction with respect to simulations with an electrostatic potential imposed according to analytic theory. This is ascribable to the smoothing of the electricfield discontinuity around the island separatrix found in the simulations. Deviations from the quadratic scaling of the perpendicular neoclassical ion flows with $\omega$ expected from the polarization-current theory are found at rotation frequencies of the order of the parallel ion streaming along the island, which is of the same order as the diamagnetic frequency for islands as wide as the poloidal ion gyroradius. These deviations are found in drift kinetic calculations (analytic and numeric) to be related to the interaction of the trapped-particle precession with the island motion. These drift kinetic results still need to be confirmed in gyrokinetic simulations with a self-consistent electrostatic potential. Moreover, at these frequencies a further contribution to the ion flow, scaling linearly with $\omega$ and appearing in the region just inside the island separatrix, is detected in both drift kinetic and gyrokinetic simulations. The origin and the role of this contribution still has to be determined, together with an eventual contribution of the electrons.

As stated above, the evolution of the tearing mode is fundamentally a multi-scale problem, in which in particular the interaction with turbulence is being explored more and more thoroughly both analytically (see e.g. Refs. $[53,54]$ ) and numerically (see e.g. Refs. $[55,56,57,58,59]$ and references therein) with respect to both the seeding process and the influence on the torque acting on the island and determining its rotation speed. Toroidal gyrokinetic simulations of turbulence in the presence of an imposed magnetic island have been carried out and the interested reader is referred to Refs. [60, 61, 62, 36] for details. It should be mentioned that self-consistent simulations of magnetic islands in the presence of electromagnetic gyrokinetic turbulence in toroidal geometry are also becoming viable now [63,64], although they still represent a tremendous computational effort. Also in this respect, simulations based on a reduced physical model, as those quoted above, allow a larger degree of flexibility in the variation of the relevant parameters and a more direct grasp on the underlying physics. 
The results presented in this paper aim to illustrate the importance of sometimes subtle effects, that can escape fluid or slab-geometry treatments, and possibly to encourage further work on the way to an overall picture which combines basic understanding with the consideration of all the necessary physical details.

\section{Acknowledgments}

Large part of the material presented in this paper has been prepared for presentations held at the $6^{\text {th }}$ IAEA Technical Meeting on Theory of Plasma Instabilities (Vienna, May 27-29, 2013), at the Lorentz-Center Workshop on Modeling Kinetic Aspects of Global MHD Modes (Leiden, December $2-6,2013$ ) and at the $1^{\text {st }}$ International Workshop on Energetic Particles and Tearing Modes (Aix-en-Provence, June 10-12, 2015). The authors would like to acknowledge the discussions and the stimulating atmosphere of these meetings.

One of the authors (D. Zarzoso) is supported by the A*MIDEX project (no. ANR-11IDEX-0001-02) funded by the "Investissements d'Avenir" French Government program, managed by the French National Research Agency (ANR). The 1st International Workshop on Energetic Particles and Tearing Modes was organized by Aix-Marseille University within the A*MIDEX framework.

A part of this work was carried out using the HELIOS supercomputer system at Computational Simulation Centre of International Fusion Energy Research Centre (IFERCCSC), Rokkasho, Japan, under the Broader Approach collaboration between Euratom and Japan, implemented by Fusion for Energy and JAEA.

\section{A Numerical solution of the drift kinetic and gyroki- netic equations in the presence of a magnetic island}

The numerical results discussed in this paper are based on the solution of the drift kinetic equation as obtained from the code HAGIS [65], augmented by a pitch-angle scattering operator including a correction to ensure momentum conservation [66] and by an magneticisland structure [30], and on the solution of the gyrokinetic equation with the code GKW [67], where a magnetic island perturbation has been implemented for both periodic [62] and non-periodic radial boundary conditions [35]. A brief account of the numerical scheme is given here. The interested reader is referred to the original publications for more details.

In drift kinetic simulations, the equation

$$
\frac{\mathrm{d} f}{\mathrm{~d} t}=\frac{\partial f}{\partial t}+\left(v_{\|} \hat{\mathbf{b}}+\mathbf{v}_{d}+\mathbf{v}_{E}\right) \cdot \frac{\partial f}{\partial \mathbf{r}}-\frac{e}{m} \frac{\mathbf{v}_{d} \cdot \nabla \Phi}{v} \frac{\partial f}{\partial v}=C(f)
$$

is solved in a toroidal geometry, including the presence of the island in the magnetic configuration, with $f$ the particle distribution function, $\mathbf{v}_{d}$ and $\mathbf{v}_{E}$ the magnetic and electric drift velocities, $e$ and $m$ the charge and the mass of the particles and $C$ is the pitchangle collision operator. The parallel electric field $E_{\|}$is set to zero since it is assumed to be immediately short-circuited by the electrons (cf. Ref. [15]). Eq.(22) is solved employing the $\delta f$ method, i.e. the distribution function is written as the sum of a time-independent analytically-known bulk term $f_{0}$ and a deviation $\delta f$, to be evaluated numerically. The equation for $\delta f$ reads

$$
\frac{\mathrm{d}(\delta f)}{\mathrm{d} t}=C(\delta f)-\mathbf{v}_{d} \cdot \nabla f_{M}-\frac{e f_{M}}{T} \mathbf{v}_{d} \cdot \nabla \Phi
$$

so that the right-hand side of Eq. (23) can be thought as the "source" causing a deviation from $f_{0}$, supposed here to be a Maxwellian $f_{M}$. No assumption is made here about the magnitude of $\delta f$ as compared to $f_{0}$, so that Eq. (23) is equivalent to Eq. (22), but the 
$\delta f$ approach is obviously computationally advantageous if $|\delta f| \ll\left|f_{0}\right|$, since $\delta f$ is to be represented numerically by an ensemble of "markers", evolving according to the equations of motion written in Boozer coordinates (these contain also the terms corresponding to the electromagnetic perturbation due to the presence of a magnetic island). The magnetic equilibrium is specified analytically for the sake of saving computational time. The unperturbed magnetic surfaces are circular and concentric. The magnetic perturbation of given helicity is assigned by choosing the mode amplitude $\tilde{\psi}$ and the mode rotation frequency $\omega$ (appearing in the helical angle $\xi=m \theta-n \varphi-\omega t$ ). Neither $\tilde{\psi}$ nor $\omega$ evolve during the run. Flux-surface averages are obtained by integrating in space between neighbouring surfaces according to

$$
\langle A\rangle=\lim _{\delta \bar{\Psi} \rightarrow 0} \frac{\int A d^{3} \mathbf{r}}{\int d^{3} \mathbf{r}} \Rightarrow \frac{1}{n}\left\langle\int A \delta f d^{3} \mathbf{v}\right\rangle \simeq \frac{\int_{\Omega-\delta \Omega}^{\Omega+\delta \Omega} A \delta f d \Gamma}{\int_{\Omega-\delta \Omega}^{\Omega+\delta \Omega} f_{0} d \Gamma} .
$$

In order to determine currents that flux-surface average to zero, a further refinement in the spatial integration is obtained by introducing smaller cells in the $\xi$-direction [42,33]. As stated in the body of the text, when a two-step approach is adopted for the determination of the bootstrap current (including the contribution of both electrons and ions), Eq. (22) is solved first for the ions, disregarding the role of ion-electron collisions, and then for the electrons, including the mean parallel ion flow as a shift in $f_{0 e}$, so that this term exactly cancels the ion contribution and the total bootstrap current is completely determined by $\delta f_{e}$ (see Ref. [33] for details).

The gyrokinetic results presented in the paper has been performed employing the Vlasov code GKW. Ampère's equation is switched off in our simulations, but a magnetic perturbation corresponding to an island of desired helicity is introduced through the specification of an appropriate parallel vector potential, see below. Fully kinetic electrons are necessary to have the correct dynamics including the motion along the perturbed magnetic field lines. The island widths considered in our simulations does never exceed several ion Larmor radii, so that the $\delta f$ approximation adopted in the code is justified. The gyrokinetic equation is solved in the form

$$
\frac{\partial g}{\partial t}+\left(v_{\|} \mathbf{b}+\mathbf{v}_{D}\right) \cdot \nabla f+\mathbf{v}_{\chi} \cdot \nabla g-\frac{\mu B}{m} \frac{\mathbf{B} \cdot \nabla B}{B^{2}} \frac{\partial f}{\partial v_{\|}}=S,
$$

where $g=f+(Z e / T) v_{\|}\left\langle A_{\|}\right\rangle F_{M}$, the source term on the right-hand side is

$$
\begin{aligned}
S= & -\left(\mathbf{v}_{\chi}+\mathbf{v}_{D}\right) \cdot\left[\frac{\nabla n}{n}+\left(\frac{v_{\|}^{2}}{v_{\mathrm{th}}^{2}}+\frac{\mu B}{T}-\frac{3}{2}\right) \frac{\nabla T}{T}\right] F_{M} \\
& -\frac{Z e}{T}\left[v_{\|} \mathbf{b}+\mathbf{v}_{D}\right] \cdot \nabla\langle\phi\rangle F_{M} .
\end{aligned}
$$

and the motion due to the perturbed electromagnetic field is expressed by the velocity

$$
\mathbf{v}_{\chi}=\frac{\mathbf{b} \times \nabla \chi}{B},
$$

which is the combination of the $E \times B$ velocity and the parallel motion along the perturbed field lines.

In the Hamada coordinates used in GKW, a magnetic perturbation of given helicity has the form (omitting for simplicity the harmonic time dependence $-i \omega t$ )

$$
A_{\|}=\tilde{A}_{\|} \exp \left[2 \pi i n\left(\zeta-s q^{\prime} \psi\right)\right]
$$

where $s$ is the parallel coordinate, $\zeta$ the generalized toroidal angle and $\psi$ the distance from the rational surface. If a Fourier representation is adopted in the radial direction,

$$
A_{\|}=e^{i k_{\zeta} \zeta} \sum_{p} A^{(p)}(s) e^{i p k_{\psi} \psi}
$$


(with $k_{\zeta}=2 \pi n$ and $k_{\psi}=k_{\zeta} q^{\prime}$ ) the corresponding amplitudes are given by

$$
A^{(p)}(s)=\tilde{A}_{\|} \frac{\sin [\pi(p+s)]}{\pi(p+s)} .
$$

In the numerical implementation, an extra smoothing factor at higher radial mode numbers of the form $\exp \left[-(p+s)^{2} / L^{2}\right]$ is introduced in order to satisfy the periodic radial boundary conditions of the sheared flux tube domain. The results are unaffected provided the scale length $L$ is chosen to be large enough. Details on the numerical implementation are provided in Ref. [62].

The possibility of imposing a magnetic island has been recently included also in the non-spectral version of GKW [35]. In this case, the implementation of the island perturbation is straightforward, a radial envelope being added to the basic helical dependence in order not to affect the boundaries of the simulation box.

\section{References}

[1] H. P. Furth, J. Killeen, and M. N. Rosenbluth, Physics of Fluids 6, 459 (1963).

[2] P. H. Rutherford, Physics of Fluids 16, 1903 (1973).

[3] F. L. Waelbroeck, Nuclear Fusion 49, 104025 (2009).

[4] B. CoppI, Physics of Fluids 8, 2273 (1965).

[5] J. F. Drake, T. M. J. Antonsen, A. B. Hassam, and N. T. Gladd, Physics of Fluids 26, 2509 (1983).

[6] B. D. Scott, A. B. Hassam, and J. F. Drake, Physics of Fluids 28, 275 (1985).

[7] R. D. Hazeltine, D. Dobrott, and T. S. Wang, Physics of Fluids 18, 1778 (1975).

[8] J. F. Drake and Y. C. Lee, Physics of Fluids 20, 1341 (1977).

[9] P. H. Rebut and M. Hugon, Plasma Physics and Controlled Fusion 33, 1085 (1991).

[10] J. W. Connor and H. R. Wilson, Physics of Plasmas 2, 4575 (1995).

[11] F. L. Waelbroeck, J. W. Connor, and H. R. Wilson, Physical Review Letters 87, 215003 (2001).

[12] A. H. Glasser, J. M. Greene, and J. L. Johnson, Physics of Fluids 18, 875 (1975).

[13] M. Kotschenreuther, R. D. Hazeltine, and P. J. Morrison, Physics of Fluids 28, 294 (1985).

[14] A. I. Smolyakov, A. Hirose, E. Lazzaro, G. B. Re, and C. J. D, Physics of Plasmas 2, 1581 (1995).

[15] H. R. Wilson, J. W. Connor, R. J. Hastie, and C. C. Hegna, Physics of Plasmas 3, 248 (1996).

[16] W. X. QU and C. J. D, University of Wisconsin Plasma Report UWPR 85-5 (1985).

[17] R. Carrera, R. D. Hazeltine, and M. Kotschenreuther, Physics of Fluids 29, 899 (1986). 
[18] O. Sauter, R. J. La Haye, Z. Chang, D. A. Gates, Y. Kamada, H. Zohm, A. Bondeson, D. Boucher, J. D. Callen, M. S. Chu, T. A. Gianakon, O. Gruber, R. W. Harvey, C. C. Hegna, L. L. Lao, D. A. Monticello, F. Perkins, A. Pletzer, A. H. Reiman, M. Rosenbluth, E. J. Strait, T. S. Taylor, A. D. Turnbull, F. Waelbroeck, J. C. Wesley, H. R. Wilson, and R. Yoshino, Physics of Plasmas 4, 1654 (1997).

[19] R. J. Lahaye, S. Günter, D. A. Humphreys, J. Lohr, T. C. Luce, M. E. Maraschek, C. C. Petty, R. Prater, J. T. Scoville, and E. J. Strait, Physics of Plasmas 9, 2051 (2002).

[20] A. Isayama, Y. Kamada, N. Hayashi, T. Suzuki, T. Oikawa, T. Fujita, T. Fukuda3, S. Ide, H. Takenaga, and K. Ushigusa, Nuclear Fusion 43, 1272 (2003).

[21] L. Urso, H. Zohm, A. Isayama, M. Maraschek, and E. Poli, Nuclear Fusion 50, 025010 (2010).

[22] N. Hayashi, T. Ozeki, K. Hamamatsu, and T. Takizuka, Nuclear Fusion 44, 477 (2004).

[23] R. J. LaHaye, A. Isayama, and M. Maraschek, Nuclear Fusion 49, 045005 (2009).

[24] E. Poli, C. Angioni, F. J. Casson, D. Farina, L. Figini, T. P. Goodman, O. Maj, O. Sauter, H. Weber, H. Zohm, G. Saibene, and M. A. Henderson, Nuclear Fusion 55, 013023 (2015).

[25] V. S. Marchenko, Nuclear Fusion 39, 1541 (1999).

[26] B. D. Scott and A. B. Hassam, Physics of Fluids 30, 90 (1987).

[27] A. I. Smolyakov, Plasma Physics and Controlled Fusion 35, 657 (1993).

[28] R. Fitzpatrick, Physics of Plasmas 2, 825 (1995).

[29] E. Poli, A. G. Peeters, A. Bergmann, S. Günter, and S. D. Pinches, Physical Review Letters 88, 075001 (2002).

[30] E. Poli, A. G. Peeters, A. Bergmann, S. Günter, and S. D. Pinches, Plasma Physics and Controlled Fusion 45, 71 (2003).

[31] O. Sauter, R. J. Buttery, R. Felton, T. C. Hender, and F. Howell, Plasma Physics and Controlled Fusion 44, 1999 (2002).

[32] A. Gude, S. Günter, and S. Sesnic, Nuclear Fusion 39, 127 (1999).

[33] A. Bergmann, E. Poli, and A. G. Peeters, Physics of Plasmas 16, 092507 (2009).

[34] M. Siccinio, E. Poli, F. J. Casson, W. A. Hornsby, and A. G. Peeters, Physics of Plasmas 18, 122506 (2011).

[35] D. Zarzoso, F. J. Casson, W. A. Hornsby, E. Poli, and A. G. Peeters, Physics of Plasmas 22, 022127 (2015).

[36] D. Zarzoso, W. A. Hornsby, E. Poli, F. J. Casson, A. G. Peeters, and S. NASR, Nuclear Fusion 55, 113018 (2015).

[37] W. A. Hornsby, M. Siccinio, A. G. Peeters, E. Poli, A. P. Snodin, F. J. Casson, Y. Camenen, and G. Szepesi, Plasma Physics and Controlled Fusion 53, 054008 (2011).

[38] A. I. Smolyakov, Sovietic Journal of Plasma Physics 15, 667 (1989). 
[39] H. R. Wilson, M. M Alexander, J. W. Connor, A. M. Edwards, D. Gates, O. Gruber, R. J. Hastie, C. C. Hegna, T. C. Hender, R. J. La Haye, L. L. Lao, A. W. Morris, C. M. Roach, E. J. Strait, T. S. Taylor, M. Valovic, and H. Zoнm, Plasma Physics and Controlled Fusion 38, A149 (1996).

[40] M. Maraschek, S. Günter, and H. Zohm, Plasma Physics and Controlled Fusion 41, L1 (1999).

[41] A. B. Mikhailovskir, Contributions to Plasma Physics 43, 125 (2003).

[42] E. Poli, A. Bergmann, A. G. Peeters, L. Appel, and S. D. Pinches, Nuclear Fusion 45, 384 (2005).

[43] E. Poli, A. Bergmann, and A. G. Peeters, Physical Review Letters 94, 205001 (2005).

[44] M. Siccinio and E. Poli, Plasma Physics and Controlled Fusion 51, 075005 (2009).

[45] K. Imada and H. R. Wilson, Plasma Physics and Controlled Fusion 51, 105010 (2009).

[46] A. Bergmann, E. Poli, and A. G. Peeters, Physics of Plasmas 12, 072501 (2005).

[47] A. B. Mikhailovskit, V. D. Pustovitov, and A. I. Smolyakov, Plasma Physics 42, 309 (2000).

[48] S. V. Konovalov, A. B. Mikhailovskit, V. S. Tsypin, and S. E. Sharapov, Doklady Physics 47, 488 (2002).

[49] J. W. Connor, F. L. Waelbroeck, and H. R. Wilson, Physics of Plasmas 8, 2835 (2001).

[50] M. James and H. R. Wilson, Plasma Physics and Controlled Fusion 48, 1647 (2006).

[51] M. James, H. R. Wilson, and J. W. Connor, Plasma Physics and Controlled Fusion 52, 075008 (2010).

[52] F. L. Waelbroeck and F. R, Physical Review Letters 78, 1703 (1997).

[53] C. J. McDevitt and P. H. Diamond, Physics of Plasmas 13, 032302 (2006).

[54] A. Sen, R. Singh, D. Chandra, P. Kaw, and D. Raju, Nuclear Fusion 49, 115012 (2009).

[55] M. Yagi, S.-I. Itoh, K. Itoh, M. Azumi, P. H. Diamond, A. Fukuyama, and T. Hayashi, Plasma and Fusion Research 2, 025 (2007).

[56] F. Militello, F. L. Waelbroeck, R. Fitzpatrick, and W. Horton, Physics of Plasmas 15, 050701 (2008).

[57] M. Muraglia, O. Agullo, S. Benkadda, X. Garbet, P. Beyer, and A. Sen, Physical Review Letters 103, 145001 (2009).

[58] A. Ishizawa and F. L. Waelbroeck, Physics of Plasmas 20, 122301 (2013).

[59] O. Agullo, M. Muraglia, A. Poyé, S. Benkadda, M. Yagi, X. Garbet, and A. Sen, Physics of Plasmas 21, 092303 (2014).

[60] E. Poli, A. Bottino, and A. G. Peeters, Nuclear Fusion 49, 075010 (2009).

[61] E. Poli, A. Bottino, W. A. Hornsby, A. G. Peeters, T. Ribeiro, B. D. Scott, and M. Siccinio, Plasma Physics and Controlled Fusion 52, 124021 (2010).

[62] W. A. Hornsby, A. G. Peeters, F. J. Casson, Y. Camenen, G. Szepesi, M. Siccinio, and E. Poli, Physics of Plasmas 17, 092301 (2010). 
[63] W. A. Hornsby, P. Migliano, R. Buchholz, L. Kroenert, A. Weikl, A. G. Peeters, D. Zarzoso, E. Poli, and F. J. Casson, Physics of Plasmas 22, 022118 (2015).

[64] W. A. Hornsby, P. Migliano, R. Buchholz, D. Zarzoso, F. J. Casson, E. Poli, and A. G. Peeters, Plasma Physics and Controlled Fusion 57, 054018 (2015).

[65] S. D. Pinches, L. C. Appel, J. Candy, S. E. Sharapov, H. L. Berk, D. Borba, B. N. Breizman, T. C. Hender, K. I. Hopcraft, G. T. A. Huysmans, and W. Kerner, Computer Physics Communications 111, 133 (1998).

[66] A. Bergmann, A. G. Peeters, and S. D. Pinches, Physics of Plasmas 8, 5192 (2001).

[67] A. G. Peeters, Y. Camenen, F. J. Casson, W. A. Hornsby, A. P. Snodin, D. Strintzi, and G. Szepesi, Computer Physics Communications 180, 2650 (2009). 